\title{
Characterizing a Tune All Bandstop Filter
}

\author{
Carles Musoll-Anguiano ${ }^{1}$, Ignacio Llamas-Garro ${ }^{1}$, Zabdiel Brito-Brito ${ }^{1}$, \\ Lluis Pradell ${ }^{1}$, Alonso Corona-Chavez ${ }^{2}$ \\ ${ }^{1}$ Signal Theory and Communications Department, Technical University of Catalonia \\ Campus Nord, 08034 Barcelona, Spain \\ 1lamasi@ieee.org \\ ${ }^{2}$ National Institute for Astrophysics, Optics and Electronics (INAOE), Puebla, 72840, Mexico
}

\begin{abstract}
In this paper a reconfigurable bandstop filter able to reconfigure central frequency, bandwidth and selectivity for fine tuning applications is presented. The reconfigurable filter topology has four poles and a quasielliptic bandstop filter response. The filter is tuned by varactor diodes placed at different locations on the filter topology. The varactors are voltage controlled in pairs due to filter symmetry for central frequency and bandwidth control. An additional varactor is placed on a crossing line to move a pair of transmission zeros, closer or farther to the filter central frequency, which tunes filter selectivity. The filter has a tuneable fractional bandwidth range from 11.51 to $15.46 \%$, a tuneable central frequency range from 1.346 to $1.420 \mathrm{GHz}$ and a selectivity tuning range from 0.37 to $0.40 \mathrm{~dB} / \mathrm{MHz}$.
\end{abstract}

Index Terms - bandstop quasi-elliptic filter, varactor diode, reconfigurable filter.

\section{INTRODUCTION}

There is an increased demand for microwave filters with advanced features that can make RF systems much more efficient and adaptable to multiple bands. Reconfigurable filters can reduce the complexity of a system avoiding the introduction of filter banks; earlier work has been primarily focused on central frequency or bandwidth control. In this paper we propose selectivity tuning adding up to central frequency and bandwidth reconfigurability. The filter described in this paper is capable of having different central frequency states with precise bandwidth control, or alternatively having different fractional bandwidths with a fixed central frequency. Moreover, the filter allows selectivity tuning for each state. The filter presented in this paper finds its application in adaptable image rejection receivers. In [1-2] reconfigurable bandstop filters using varactor loaded resonators are presented, these filters can reconfigure their central frequency, but do not take into account the significant increase in bandwidth for the different filter states. The bandstop filter presented in [3] uses a thermal actuator as tuning element; the central frequency can be tuned from 5.75 to $6.09 \mathrm{GHz}$, without bandwidth control. In [4] a bandstop filter using electromagnetic bandgap periodic structures on coplanar transmission lines is shown; MEMS bridges are used as tuning elements, the filter central frequency varies from 17 to $22.5 \mathrm{GHz}$. The bandwidth progressively varies for different values of central frequency. The bandstop filter in [5] uses MEMS switches; the filter is designed with microstrip transmission lines including radial stubs, the filter has a margin of reconfiguration from 8 to $15 \mathrm{GHz}$, however the bandwidth presents arbitrary values at each central frequency. The bandstop filter in [6] uses quarterwavelength stubs and MEMS switches, the filter is able to produce central frequencies in the range from 39 to 58 $\mathrm{GHz}$ with different bandwidths. The bandstop filters in [78] use PIN diodes to control filter central frequency, meanwhile varactor diodes provide a continuous bandwidth reconfiguration at given frequency. The filter has a central frequency range from 0.5 to $2 \mathrm{GHz}$ and bandwidths in the range from 30 to $42 \%$. In [9] a switchable bandstop filter with two different central frequencies is presented, the filter topology allows precise control over the design parameters frequency and bandwidth, achieved by choosing resonator sections switched by PIN diodes. The microstrip bandstop filter demonstrated in this paper allows tuning selectivity, bandwidth and central frequency. and is to our knowledge the first filter able to tune its selectivity. This paper is divided in five sections, section II contains a discussion of the proposed filter topology, describing how the filter design parameters were controlled. Section III describes how the filter was implemented, including bias circuitry. Section IV contains a discussion of measured filter responses. Finally section $\mathrm{V}$ gives an overall conclusion of this work.

\section{TUNE ALl BANDSTOP FILTER TOPOLOGY}

Fig. 1 shows the proposed filter topology which consists of a main transmission line with four capacitive coupled quarter-wavelength resonators grounded on one side. These resonators are coupled to the main transmission line through varactor diodes destined to control the fractional 
bandwidth of the filter $\left(C_{2}, C_{3}, C_{4}\right.$ and $\left.C_{5}\right)$. Filter central frequency is controlled by four varactor diodes located at the opposite end of the resonators $\left(C_{6}, C_{7}, C_{8}\right.$ and $\left.C_{9}\right)$. The crossing line containing $C_{1}$ in the middle in Fig. 1 introduces a single pair of transmission zeros. The position of these transmission zeros is controlled by $C_{1}$ destined to tune filter selectivity for a given central frequency and bandwidth.

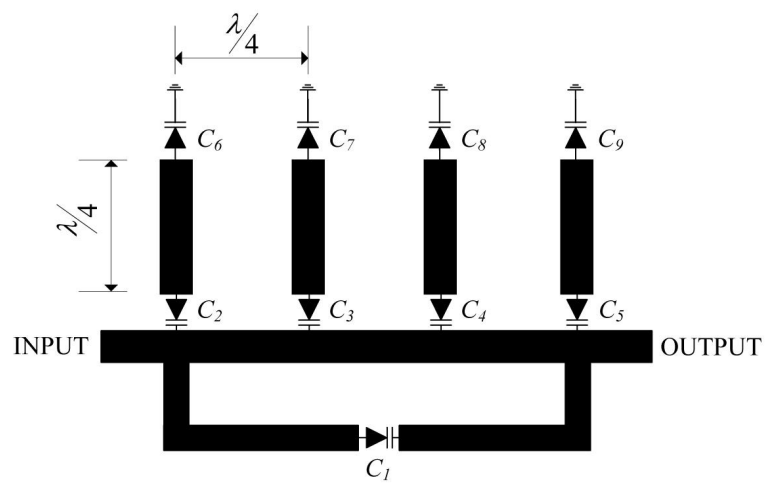

Fig. 1. Tune all bandstop filter topology.

\section{IMPLEMENTATION}

This section describes filter layout, including the bias lines used to polarize the varactor diodes, details of the surface mount components used and substrate characteristics (see Fig. 4). The filter was defined lithographically on a Rogers Duroid substrate $\left(\varepsilon_{\mathrm{r}}=2.2\right.$ and $\tan \delta=0.0009$ ). The RF choke consists of a $177 \mathrm{nF}$ inductor from Tyco Electronics with a self resonance at $1.7 \mathrm{GHz}$. The choke inductor has been modeled as a $177 \mathrm{nF}$ inductor in parallel with a $50 \mathrm{fF}$ capacitor. The simulated choke inductor isolation using ADS along the band of interest is shown in Fig. 2. Although the initial filter central frequency is $1.5 \mathrm{GHz}$, the inductor isolation is better than $-35 \mathrm{~dB}$ within the filter operation frequency range. Due to the high isolation of the choke inductor, the microwave signal is not influenced by the DC ports used to provide bias to the active devices.

In order to prevent a short circuit between the main transmission line and the crossing line, a DC block has been inserted on the crossing line shown in Fig. 3. The DC block used was a $1 \mathrm{nF}$ capacitor.

Three DC sources in Fig. 3 were used to tune the three filter design parameters. $V_{1}$ controls filter selectivity, $V_{2}$ controls filter bandwidth and $V_{3}$ controls filter central frequency. These DC sources supply the reverse voltage needed to bias the varactor diodes. The grey zone in Fig. 3 has positive polarization and the black one has negative polarization. The varactors were biased using a voltage ranging from 0 to $20 \mathrm{~V}$ according to the manufacturer's data sheet

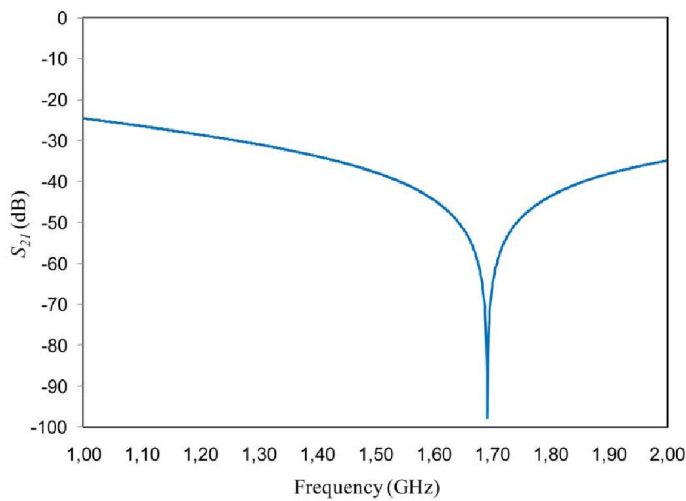

Fig. 2. Simulated RF choke response.

MACOM varactor diodes MA46470-276 were used for $C_{1}, C_{2}, C_{3}, C_{4}$ and $C_{5}$, and MA4ST402-287 were used for $C_{6}, C_{7}, C_{8}$ and $C_{9}$. The MA46470-276 diodes have a capacitance range from 0.3 to $1.8 \mathrm{pF}$ and the MA4ST402287 diodes have a capacitance range from 3.86 to 86.29 $\mathrm{pF}$. The filter layout including bias circuitry and locations of the surface mount components is shown in Fig. 4. A photograph of the fabricated filter is shown in Fig. 5.

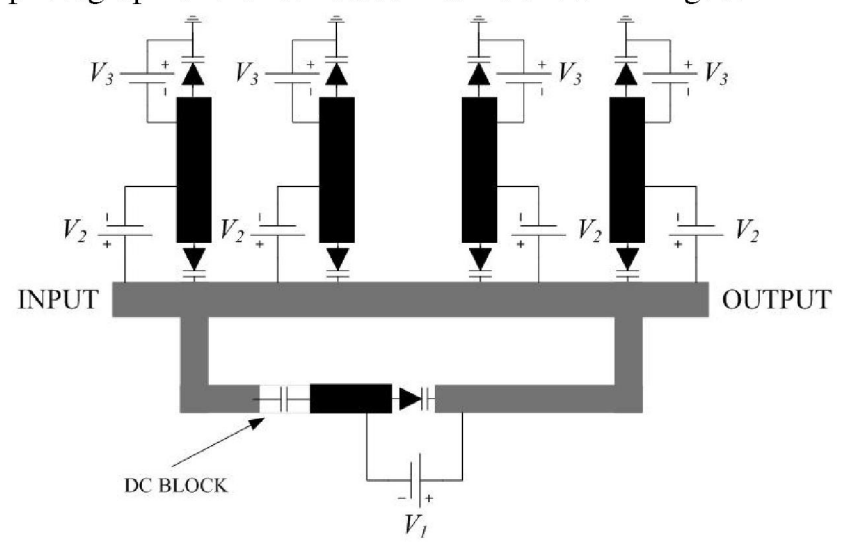

Fig. 3. Circuit bias and DC block location.

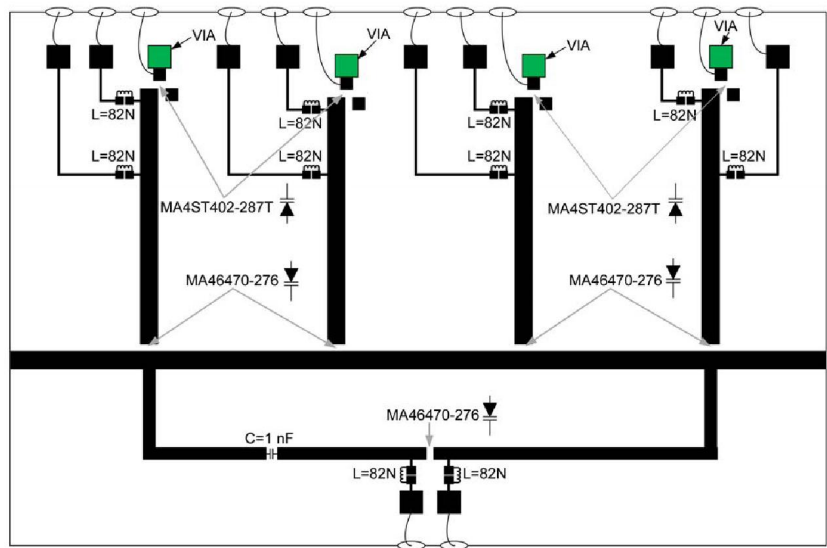

Fig. 4. Filter layout. 


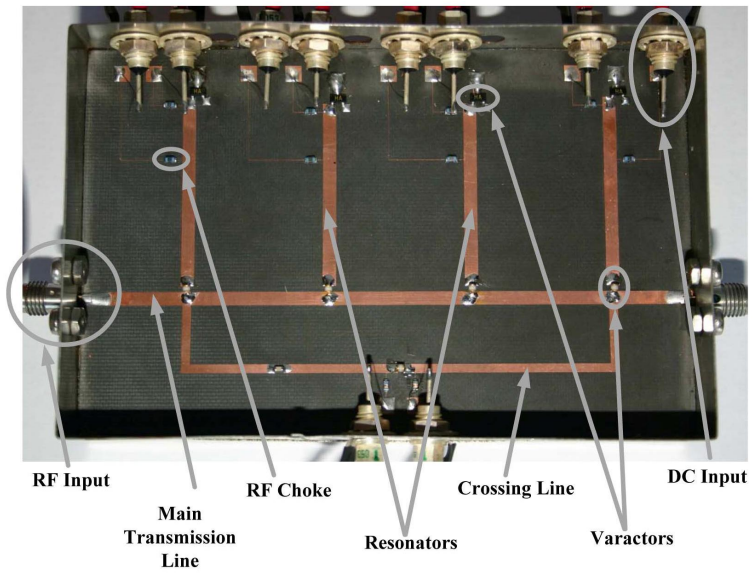

Fig. 5. Photograph of the reconfigurable bandstop filter.

\section{MEASUREMENTS}

This section contains the measurements obtained from the proposed filter. The section is divided in three parts, section $A$ shows the results obtained when selectivity is tuned for a fixed central frequency and bandwidth. Section $B$ describes the results for bandwidth tuning maintaining a fixed central frequency and selectivity. Finally section $C$ shows the results for central frequency tuning maintaining bandwidth and selectivity to a fixed value. All measurements were done after a short, open, load, thru calibration, setting the measurement reference plane at the SMA coaxial connectors used to interface the filter with the measurement equipment. Scattering parameters were measured using an Agilent 8510C network analyzer, DC bias was supplied by two Promax FAC-662B power supplies.

\section{A. Selectivity Tuning}

In Fig. 6 measurements on selectivity tuning are shown. First the bandwidth and central frequency are fixed, and then different values of selectivity are taken by varying the capacitance of varactor diode $C_{1}$ in Fig. 1 . To calculate selectivity a zoom has been taken to the squared region in Fig. 6, shown in Fig. 7. To calculate the selectivity for each filter state, we have taken the slope at the most linear part of the filter response in the passband to stopband transition region. The most linear region was found to be between -5 and $-10 \mathrm{~dB}$ as shown in Fig. 7. The varactor diode $C_{1}$ is reverse-biased by $V_{1}$ to produce a selectivity variation from 0.37 to $0.40 \mathrm{~dB} / \mathrm{MHz}$ while $V_{2}$ and $V_{3}$ are fixed to 2 and $20 \mathrm{~V}$ respectively. The reverse voltage of $V_{l}$ ranges from 2 to $20 \mathrm{~V}$. A higher variation in selectivity was observed when the bias voltage values were in the range from 15 to $20 \mathrm{~V}$. Within these voltage bias values the capacitance of the varactor diode is slighter. Therefore, as the reverse voltage increases, the capacitance values decrease, and the filter selectivity for the filter becomes lower. Selectivity tuning is independent from the other design parameters. The insertion loss for the filter in the passband varies between 1 to about $5 \mathrm{~dB}$, as shown in Fig. 6.

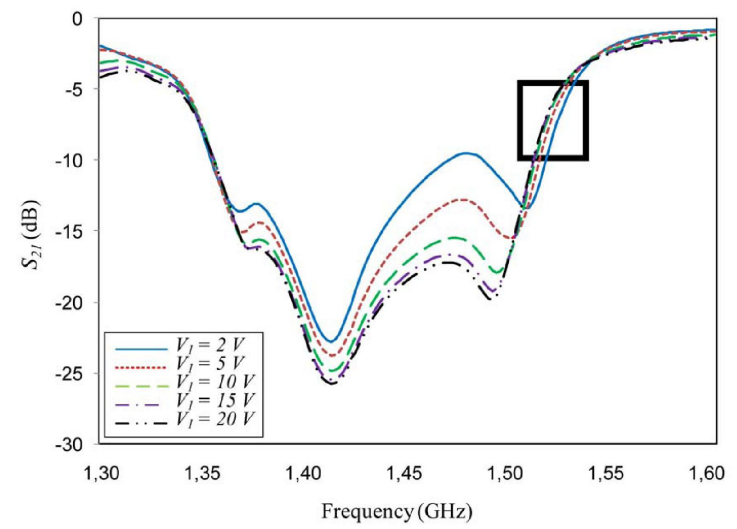

Fig. 6. Selectivity tuning.

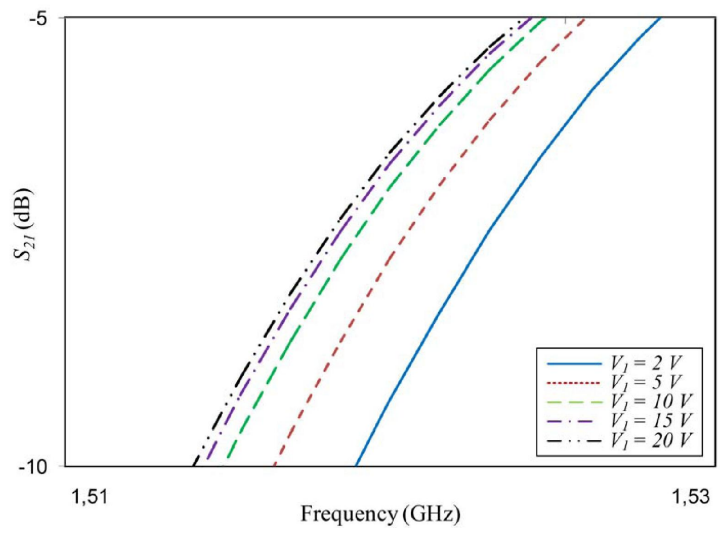

Fig. 7. Enlargement of the square region in Fig. 6.

\section{B. Bandwidth Tuning}

To tune filter bandwidth, $V_{2}$ and $V_{3}$ were varied together, meanwhile $V_{1}$ is fixed to $2 \mathrm{~V}$. $V_{2}$ modifies resonator capacitive coupling from the main transmission line, thus adjusts the bandwidth of the filter. This produces a variation in filter central frequency. The DC source $V_{3}$ adjusts the electrical length of the resonators to maintain the same central frequency for the filter. Fig. 8 shows bandwidth tuning of the filter at a central frequency of $1.41 \mathrm{GHz}$.

The tunable fractional bandwidth for the filter ranges from 11.51 to $15.46 \%$. The insertion loss in the passband ranges from 1 to approximately $5 \mathrm{~dB}$. 


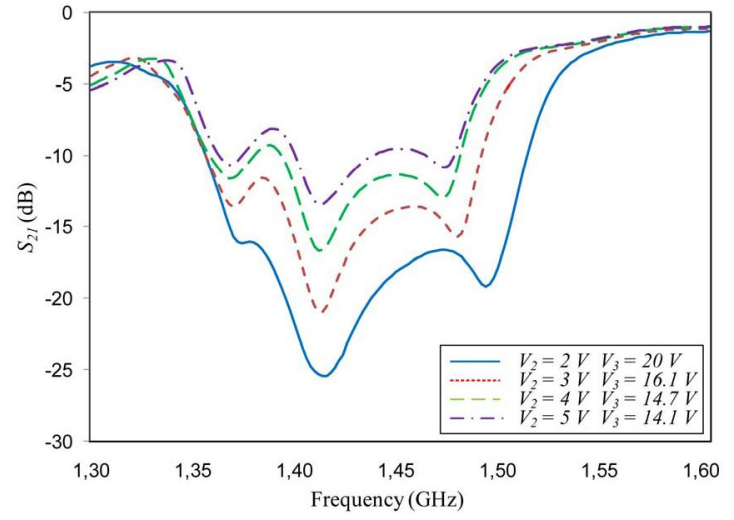

Fig. 8. Bandwidth tuning.

\section{Central Frequency Tuning}

To obtain the tunable central frequency measurements, the reverse voltage $V_{1}$ was fixed to $2 \mathrm{~V}, V_{2}$ was fixed to 20 $\mathrm{V}$ and $V_{3}$ varies between 16 and $21 \mathrm{~V}$. The varactor diodes $C_{6}, C_{7}, C_{8}$ and $C_{9}$ were reverse biased by $V_{3}$ which adjusts the resonator electrical lengths to produce the reconfigurable filter central frequency shown in Fig. 9.

A central frequency variation of $72 \mathrm{MHz}$ was obtained. The central frequency is tuned from 1.346 to $1.420 \mathrm{GHz}$ using bias voltages of 16 and $21 \mathrm{~V}$ respectively. The insertion loss in the passband ranges from 5 to $9 \mathrm{~dB}$.

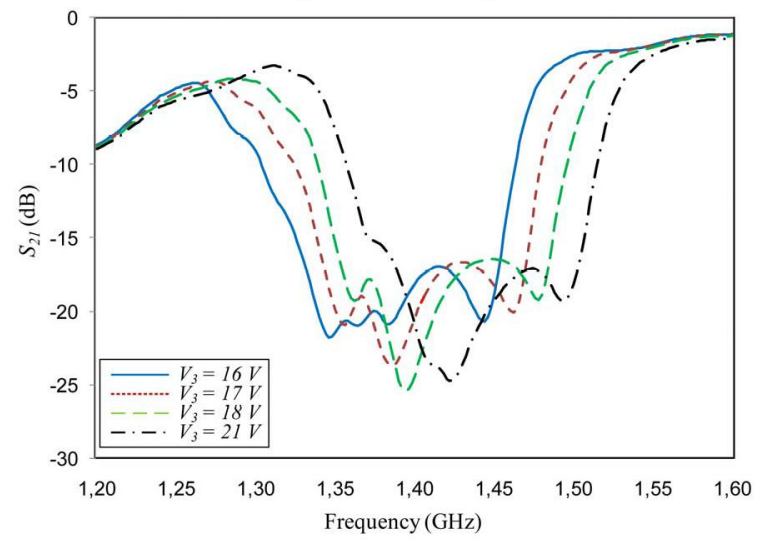

Fig. 9. Central frequency tuning.

\section{CONCLUSIONS}

A reconfigurable bandstop filter with a pair of transmission zeros has been designed implemented and measured. Selectivity tuning for the filter has been obtained by varying the capacitance of a varactor diode situated on the crossing line that produces the cross coupled circuit. Bandwidth tuning has been controlled by varactor diodes used to couple resonators to the main transmission line. The central frequency of the filter is controlled by varactor diodes placed at the end of quarterwavelength resonators. The filter topology presented in this paper is able to adjust all filter design parameters continuously, and can be perfectly adjusted to produce a fractional bandwidth range from 11.51 to $15.46 \%$, a central frequency range from 1.346 to $1.420 \mathrm{GHz}$ and a selectivity tuning range from 0.37 to $0.40 \mathrm{~dB} / \mathrm{MHz}$.

\section{ACKNOWLEDGEMENT}

This work has been financed by research project TEC2007-65705/TCM from the Spanish Ministry of Education and Culture, and research project 2006ITT10005 from AGAUR- Generalitat de Catalunya.

\section{REFERENCES}

[1] Hunter, I.C.; Rhodes, J.D.; "Electronically Tunable Microwave Bandstop Filters" IEEE Transactions on Microwave Theory and Techniques, Vol. 30, No. 9, Sept. 1982, pp. $1361-1367$.

[2] Chandler, S.R; Hunter, I.C; Gardiner, J.G.; "Active varactor tunable microwave filters" $23 \mathrm{rd}$ European Microwave Conference, Oct. 1993, pp. $244-245$.

[3] Yan, W. D.; Mansour, R. R.; "Compact Tunable Bandstop Filter Integrated with Large Deflected Actuators" in IEEE/MTT-S International Microwave Symposium, 3-8 Jun. 2007, pp. $1611-1614$.

[4] Karim, M.F.; Liu, A.Q.; Yu, A.B.; Alphones, A.; 'MEMSbased tunable bandstop filter using electromagnetic bandgap (EBG) structures" Asia-Pacific Microwave Conference, Vol. 3, 4-7 Dec. 2005, 4 pp.

[5] Guizhen Zheng, John Papapolymerou, "Monolithic reconfigurable bandstop filter using RF MEMS switches" International Journal of $R F$ and Microwave ComputerAided Engineering, Vol. 14, No. 4, Jul. 2004, pp. 373 - 382.

[6] Takacs, A.; Neculoiu, D.; Vasilache, D.; Muller, A.; Pons, P.; Aubert, H.; Plana, R.; "Tunable MEMS Filters for Millimeter Wave Applications", International Semiconductor Conference, Vol. 1, Sept. 2006, pp. 115 118

[7] B.E. Carey-Smith; P.A. Warr; "Broadband-configurable bandstop-filter design employing a composite tuning mechanism" IET Proc. Microwaves, Antennas and Propagation, Vol. 1, No. 2, Apr. 2007, pp. 420- 426.

[8] Carey-Smith, B.; Warr, P.A.; "Broadband configurable bandstop filter with composite tuning mechanism" Electronics Letters, Vol. 40, No. 25, 9 Dec. 2004, pp. 1587 $-1589$.

[9] Zabdiel Brito-Brito, Ignacio Llamas-Garro, Lluis PradellCara, Alonso Corona-Chavez., "Microstrip Switchable Bandstop Filter using PIN Diodes with Precise Frequency and Bandwidth Control", 38th European Microwave Conference, Amsterdam, The Netherlands, 28-30 Oct. 2008, pp. $1707-1710$ 